\title{
Knockdown of Ran GTPase expression inhibits the proliferation and migration of breast cancer cells
}

\author{
CHENYI SHENG $^{1 *}$, JIAN QIU $^{1 *}$, YINGYING WANG $^{2}$, ZHIXIAN HE $^{1}$, HUA WANG $^{1}$, QINGQING WANG $^{1}$, \\ YEQING HUANG ${ }^{3}$, LIANXIN ZHU ${ }^{4}$, FENG SHI ${ }^{1}$, YINGYING CHEN ${ }^{2}$, \\ SHIYAO XIONG ${ }^{1}$, ZHEN XU ${ }^{2}$ and QICHAO NI ${ }^{1}$ \\ ${ }^{1}$ Department of General Surgery, Affiliated Hospital of Nantong University; ${ }^{2}$ Surgical Comprehensive Laboratory, \\ Medical School of Nantong University, Nantong, Jiangsu 226001; ${ }^{3}$ Department of Pathology, Affiliated Cancer Hospital of \\ Nantong University, Nantong, Jiangsu 226361; ${ }^{4}$ Department of Surgical Oncology, Lu'an People's Hospital Tumor Center, \\ The Lu'an Affiliated Hospital of Anhui Medical University, Lu'an, Anhui 237000, P.R. China
}

Received February 12, 2017; Accepted April 16, 2018

DOI: $10.3892 / \mathrm{mmr} .2018 .8952$

\begin{abstract}
Breast cancer is the second leading cause of cancer-associated mortality in women worldwide. Strong evidence has suggested that Ran, which is a small GTP binding protein involved in the transport of RNA and protein across the nucleus, may be a key cellular protein involved in the metastatic progression of cancer. The present study investigated Ran gene expression in breast cancer tissue samples obtained from 140 patients who had undergone surgical resection for breast cancer. Western blot analysis of Ran in breast cancer tissues and paired adjacent normal tissues showed that expression of Ran was significantly increased in breast cancer tissues. Immunohistochemistry analyses conducted on formalin-fixed paraffin-embedded breast cancer tissue sections revealed that Ran expression was associated with tumor histological grade, nerve invasion and metastasis, vascular metastasis and Ki-67 expression (a marker of cell proliferation). Kaplan-Meier survival analysis showed that increased Ran expression in patients with breast cancer was positively associated with a poor survival prognosis. Furthermore, in vitro experiments demonstrated that highly migratory MDA-MB-231 cancer cells treated with Ran-si-RNA (si-Ran), which knocked down expression of Ran, exhibited decreased motility in trans-well migration and wound healing assays. Cell cycle analysis of Ran knocked down MDA-MB-231 cells implicated Ran in cell cycle arrest and the inhibition of proliferation. Furthermore, a starvation and re-feeding (CCK-8) assay was performed, which indicated that Ran regulated breast cancer cell proliferation.
\end{abstract}

Correspondence to: Professor Qichao Ni, Department of General Surgery, Affiliated Hospital of Nantong University, 20 West Temple Road, Nantong, Jiangsu 226001, P.R. China

E-mail: nqcuser@163.com

${ }^{*}$ Contributed equally

Key words: RanGTPase, breast cancer, proliferation, migration
Taken together, the results provide strong in vitro evidence of the involvement of Ran in the progression of breast cancer and suggest that it could have high potential as a therapeutic target and/or marker of disease.

\section{Introduction}

Breast cancer is the most commonly diagnosed cancer in women worldwide and is a leading cause of cancer-related mortality in developed countries (1). According to recent research, the prevalence of breast cancer has risen such that it is now the second leading killer of women among all cancers (2). As a disease with a complex, multifarious genetic and biochemical background, the precise mechanisms of breast carcinoma development remain somewhat unclear. Hence, there is an urgent need for the identification of prognostic and predictive biomolecular and genetic markers that are involved in the progression of breast cancer, in order to inform the design of more effective intervention therapies.

Ras-related nuclear protein (Ran) is a small ras-related GTPase that is recognized as having a number of important roles in a range of human cellular functions. Most notably, Ran is essential for proper nucleocytoplasmic transport, mitosis and nuclear envelope formation (3-6). It mediates nucleocytoplasmic transport through the association of RanGTPase with the importin- $\beta$ family of importin- $\beta 1$ in the cell nucleus, which is necessary for the release of importin-a-cargo (7). Expression of the Ran interfering mutant has been observed to inhibit the nuclear import of STAT3 (8). Within cells, Ran alters between two conformations, Ran-GTP and Ran-GDP. Ran-GTP is localized in the nucleus of cells whereas Ran-GDP is found exclusively in the cytosol. This GTP/GDP switch in conformation allows Ran to migrate across nuclear pore complexes, transporting proteins and mRNA along with it (9-11).

Recently, the research focus on Ran has shifted to studying abnormalities arising from its dysregulation and subsequent genetic instability and these may be linked to the progression of cancers (12). Inhibition of Ran expression in several tumor cell lines has been shown to cause abnormal mitotic spindle formation, mitochondrial dysfunction, and apoptosis $(13,14)$. 
It is now well established that Ran is overexpressed in various cancers and that this overexpression is correlated with increased aggressiveness of the cancer cells both in vitro and in vivo (15-17). In ovarian cancer, high expression of Ran is associated with high-grade (advanced) tumors, local invasion and tumor metastasis, suggesting it as a promising prognostic indicator of poor survival (18). High expression of Ran GTPase has additionally been associated with local invasion and metastasis of human clear cell renal cell carcinoma (19). Furthermore, Ran overexpression induces a metastatic phenotype through deregulation of effector proteins with known oncogenic effects, such as Aurora A (20), the microtubule associated protein HURP (21), and BRCA1 (22).

Loss of Ran in normal cells confers minimal effects, whereas downregulation in cancer cells is associated with mitotic defects and increased apoptosis (23). The decreased survival of cancer patients may be linked with the overexpression of Ran, which is known to promote metastasis (15). Ectopic expression of Ran has been observed to enhance invasion and induce epithelial mesenchymal transition (EMT) in non-small cell lung cancer (NSCLC) cells, through the activation of PI3K-AKT signaling (24). Thus, Ran may be a potential target for NSCLC therapeutic intervention.

Last but not least, the GTPase activity of Ran is also required for efficient metastasis (15). RanGTP levels can be regulated by serum growth factors, and in particular by the growth factor HRG. Increased RanGTP levels have been associated with increased cell transformation and tumorigenicity (17). Therefore, there exists an opportunity to develop Ran inhibitors that selectively induce apoptosis in malignant cells as a potential future therapy for the treatment of a range of human cancers.

Against this background, Ran plays an important role in cancer development and progression. It is overexpressed in various cancers with prognostic significance, and its overexpression is correlated with increased aggressiveness of the cancer cells in vitro and in vivo (23). Ran has been shown to be a promising cancer therapeutic target. The present study focused on the analysis of the expression of Ran in breast cancer patient tissue samples and cell lines and investigated its relationship with clinicopathological features of the disease in order to determine its prognostic value for breast cancer patient survival. Furthermore, we investigated the possible role of Ran in the proliferation, invasion and metastasis of breast cancer cell lines. We sought to determine whether Ran could be a novel therapeutic target for breast cancer.

\section{Materials and methods}

Patients and tissue samples. Breast cancer tissue sections and adjacent normal tissue samples were obtained from 140 patients that had had all undergone breast surgical resection at the Department of General Surgery of the Affiliated Hospital of Nantong University, China, between April 2002 and May 2010. The patients recruited to the study had not previously undergone treatment with chemotherapy or radiotherapy prior to collection of their tissue samples. The duration of the follow-up period is nearly ten years. Histological diagnoses were formulated by two pathologists independently, The TNM system of tumor staging was used to classify the histological grade of the tumors, in accordance with World Health Organization guidelines. Clinicopathological characteristics were noted in all cases. Corresponding patient clinical histories were obtained from follow-up visits to the clinic, and by telephone contact. We have obtained an approval from Institutional Review Board (IRB) and Ethical Review Board (ERB) of the Affiliated Hospital of Nantong University. Written informed consent was obtained from each patient for tissue collection and molecular analysis.

Antibodies. The antibodies used in the immunohistochemical studies included: Goat anti-Ran specific antibody (sc-1,156; Santa Cruz Biotechnology, Santa Cruz, CA, USA), anti-Ki-67 (AB9260, 1:100; Millipore, Billerica, MA, USA). Antibodies used for western blot analysis included: Anti-Ran (1:500), anti-proliferating cell nuclear antigen (PCNA $(1: 1,000)$, anti-cyclin E, anti-cyclin A, anti-E-cadherin, anti-vimentin, and anti-GAPDH (sc-7196, 1:1,000)), all of which were purchased from Santa Cruz Biotechnology.

Western blot analysis. Prior to immunoblotting analysis, cells were washed with ice-cold PBS, resuspensed in 2 lysis buffer (50 mM Tris-HCl, $120 \mathrm{mM} \mathrm{NaCl}, 0.5 \%$ Nonidet P-40, $100 \mathrm{mM}$ $\mathrm{NaF}, 200 \mathrm{mM} \mathrm{Na} \mathrm{VO}_{4}$, and protease inhibitor mixture), and incubated for $20 \mathrm{~min}$ at $48^{\circ} \mathrm{C}$ while rocking. Lysates were cleared by centrifugation $\left(10 \mathrm{~min} 12,000 \mathrm{rpm}, 48^{\circ} \mathrm{C}\right)$ and $50 \mathrm{mg}$ total protein was resolved by SDS-PAGE and transferred on to a polyvinylidene fluoride (PVDF) membrane (Immbilon; Millipore). The membranes were first blocked and then incubated with the primary antibodies described below for $2 \mathrm{~h}$ at room temperature. The secondary antibodies were visualized using LumiGLO Regent and Peroxide (Cell Signaling Technology, Danvers, MA, USA). The optical density on the film was measured with a computer imaging system (Imaging Technology, Ontario, Canada).

Immunohistochemical staining. In brief, tissue slices were dewaxed in xylene, rehydrated with graded solutions of ethanol in water, and the endogenous peroxidase activity was blocked by steeping in $3 \%$ methanolic peroxide for $20 \mathrm{~min}$. The tissue sections were then heated to $121^{\circ} \mathrm{C}$ in an autoclave for $10 \mathrm{~min}$ in $0.1 \mathrm{M}$ citrate buffer ( $\mathrm{pH}$ 6.0) to retrieve the antigen. After rinsing three times in phosphate-buffered saline (PBS, $\mathrm{pH}$ 7.2), the sections were incubated with Ran antibody (diluted 1:200) and $\mathrm{Ki}-67$ antibody (diluted 1:500) for $3 \mathrm{~h}$ at room temperature. After washing with PBS, the peroxidase reaction was visualized by incubation with DAB (comprising of $0.1 \%$ phosphate buffer solution, $0.02 \%$ diaminobenzidine tetrahydrochloride, and $3 \% \mathrm{H}_{2} \mathrm{O}_{2}$ ). Finally, the sections were counter stained with hematoxylin, dehydrated with graded alcohol solutions, and covers lipped after rinsing in water.

Immunohistochemical evaluation. Immunostained sections were evaluated at random by three independent blinded observers, using a Leica fluorescence microscope (Leica Microsystems GmbH, Wetzlar, Germany). The semi-quantitative analysis of tissue sections following staining with Ran, was based on nuclear and cytoplasmic staining. Five views were chosen per slide, and at least 500 cells were counted per high power field of view. To evaluate the immunoreaction of Ran, 
staining intensity was estimated in comparison to a control (The control is the staining of normal breast tissue, which is a negative staining control), and scored as follows: Negative staining (0), weak staining (1), moderate staining (2), or strong staining (3). Using the Ran expression ratio, the intensity of immunostaining in each tumor section was semi-quantitatively assessedas: Negative (1), low (2), moderate (3), or high (4) expression, using the following scale: $10 \%$ of cells stained (1), $10-35 \%$ (2), $35-50 \%$ (3), and $>50 \%$ of cells stained (4). On this basis, the samples were considered to express high levels of Ran if the total score was $\geq 6$, and low levels of Ran if the score was $<6$. In the same way, the Ki-67 immunoreactivity of tumor sections was classified into a high expression group and a low expression group.

Cell culture and cell cycle analysis. Three human breast cancer cell lines: MCF-7, SKBR-3 and MDA-MB-231, obtained from the cell bank of the Chinese academy of sciences, Shanghai, China, were used in this study. All the cell lines were maintained in DMEM (Gibco-BRL, Grand Island, NY, USA) supplemented with $10 \%$ heat-inactivated fetal bovine serum (FBS), $2 \mathrm{mM}$ L-glutamin and $100 \mathrm{U} / \mathrm{ml}$ penicillin, at $37^{\circ} \mathrm{C}$ and atmospheric conditions of $5 \% \mathrm{CO}_{2}$. Starvation and re-feeding of cells was used to imitate the cell cycle. First, we used DMEM medium without fetal bovine serum to incubate MCF-7, SKBR-3 and MDA-MB-231 cells for $48 \mathrm{~h}$ to synchronize the cells, and then changed to the complete medium. We harvested cells rapidly at various time-points and immediately fixed them in $70 \%$ ethanol for at least $24 \mathrm{~h}$ at $-20^{\circ} \mathrm{C}$, prior to incubation with $1 \mathrm{mg} / \mathrm{ml}$ RNase A for $20 \mathrm{~min}$ at $37^{\circ} \mathrm{C}$. Subsequently, cells were stained with $0.5 \%$ Tween-20, propidium iodide (PI, $50 \mathrm{mg} / \mathrm{ml}$ ) in PBS, and analyzed using a Becton-Dickinson flow cytometer BD FACScan (BD Biosciences, San Jose, CA, USA), as well as Cell Quest acquisition and analysis programs.

RNA interference of Ran. Small interference RNAs (si-RNAs) were designed and chemically synthesized by Genechem (Shanghai, China). The Ran specific si-RNA target sequences were: Ran-si-RNA\#0 (si0), 5'ACAGTATGAGCACGACT TA3'; Ran-si-RNA\#1 (si1), 5'CCCTAACTTGGAATTTGT T3'; and Ran-si-RNA\#2 (si2), 5'GGATATTAAGGACAGGAA A3'. The nonspecific scrambled si-RNA sequence (ctrl) was 5'-GTCATTTGACTGGTGAATT-3'. Cells were transfected with $100 \mathrm{nmol} / \mathrm{l}$ of si-RNA duplexes using Lipofectamine Plus (Invitrogen; Thermo Fisher Scientific, Inc., Waltham, MA, USA), according to the manufacturer's protocol.

\section{Cell proliferation assay}

(1). EdU pulse chase incorporation. MDA-MB-231 cells grown on 96-well plate were cultured in triplicate in 96-well plates at a density of $1 \times 10^{3}$ for $12 \mathrm{~h}$. Then, cells were labeled with $30 \mu \mathrm{M}$ 5-ethynyl-2'-deoxyuridine (Ribobio, Guangzhou, China) for $1 \mathrm{~h}$ at $37^{\circ} \mathrm{C}$. The cells were fixed with $50 \mu \mathrm{l} 4 \%$ formaldehyde for $30 \mathrm{~min}$ and $0.5 \%$ Triton X-100 for $20 \mathrm{~min}$ for permeabilization. One hundred microliters 1x Apollo reaction cocktail was added to each well for 30 min after washing with PBS thrice. Then, cells were stained with $100 \mu$ l Hoechst 33342 for $30 \mathrm{~min}$ and washed with PBS. The stained cells were visualized under a fluorescent microscope (Olympus Corporation, Tokyo, Japan) and counted with Photoshop (Adobe Systems,
Inc., San Jose, CA, USA). The incorporation rate represents EdU fluorescence against DNA content.

(2). Cell Counting Kit-8 (Dojindo Laboratories, Kumamoto, Japan) was employed to assess cell proliferation. In brief, cells were seeded on a 96-well cell culture cluster (Corning Inc., Corning, NY, USA) at a concentration of $2 \times 10^{4} /$ well in a volume of $100 \mu \mathrm{l}$, and grown overnight. Cell Counting Kit-8 (Dojindo) reagents were then added, incubated for $2 \mathrm{~h}$ at $37^{\circ} \mathrm{C}$, and absorbance was quantified on an automated plate reader. Each experiment was performed in triplicate and repeated at least three times.

Transwell migration assays. The cells that had been transfected with the Ran-si-RNAs were starved overnight in DMEM media with $0.1 \%$ FBS and then trypsinized and resuspended in DMEM containing $0.1 \%$ bovine serum albumin. The cell suspensions were then added to the top chambers of the Transwells (Corning Inc, Corning, USA, $8 \mathrm{~mm}$ pore size) in 24-well plates, and DMEM with $10 \%$ FBS was added to the bottom chambers. After incubation overnight, the cells that remained in the top chamber (nonmigrated) were removed, and the cells in the bottom chamber (migrated) were fixed and stained with crystal violet to visualize the nuclei. The number of migrating cells was counted in five fields of view at $x 2,000$ magnification, and the mean number of cells per chamber was determined. All experiments were conducted in triplicate and repeated twice.

Wound healing assays. Cells were seeded to nearly complete confluence in a monolayer in 6-well plates. After transfected $48 \mathrm{~h}$, cell were serum starved for $12 \mathrm{~h}$. Then scratching the monolayer with a $10 \mathrm{ml}$ pipette tip, cells were washed with PBS, cultured in 5\% FBS-DMEM at 5\% $\mathrm{CO}_{2}$ and $37^{\circ} \mathrm{C}$ and photographed under 20 objective lens every $3 \mathrm{~h}$ by inverted Leica phase-contrast microscope (Leica DFC 300 FX).

Statistical analysis. All data were analyzed using SPSS 17.0 software package (SPSS, Chicago, IL, USA). The statistical significance of correlations between cellular expression of Ran and Ki-67 was analyzed using the Spearman's correlation co-efficient and the clinicopathological features of the breast cancer tissue samples were analyzed using the $\chi^{2}$ test. Kaplan-Meier analysis was undertaken to generate survival curves and these were compared using the log-Rank test. Multivariate analysis was performed using Cox's proportional hazards model, to identify significant differences between groups. For comparison of two groups, two-tailed unpaired t-test was used. For comparison of more than two groups, one-way analysis of variance followed by Tukey's post hoc test was used. $\mathrm{P}<0.05$ was considered to indicate a statistically significant difference.

\section{Results}

Expression of Ran and Ki-67 in human malignant breast tissues and breast cancer cell lines. The analysis of Ran expression in breast cancer tissue samples by western blotting showed that Ran is over expressed in breast cancer cells, but only expressed at low or undetectable levels in the cells of paired adjacent normal tissues (Fig. 1A and B). Among the 
A

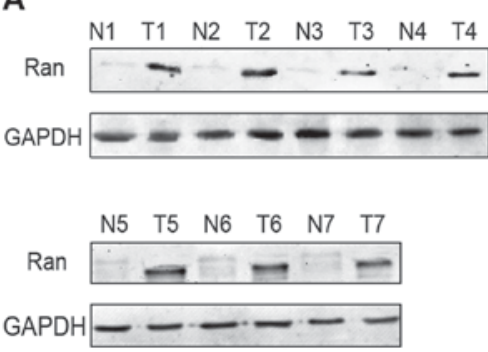

C

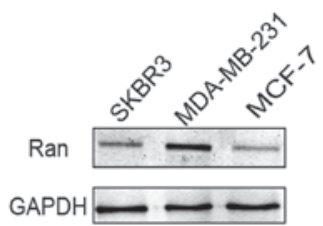

B
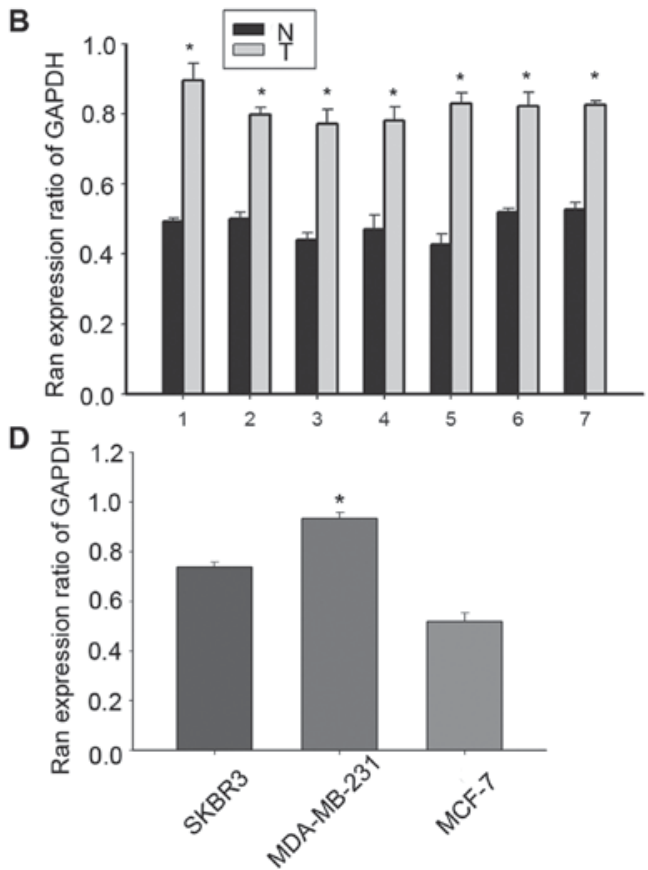

Figure 1. Expression of Ran in breast cancer tissues. (A) The expression of Ran in seven paired adjacent normal tissues (N1-N7) and breast carcinoma tissues (T1-T7), assessed by western blotting. The data from each time-point were derived from three independent experiments. (B) Expression levels of Ran in breast cancer tissues and paired adjacent normal tissues, assessed by density photometry. (C) Expression of Ran in three breast cancer cell lines, analyzed by western blotting. The data from each time-point were derived from three independent experiments. (D) Expression levels of Ran protein in three breast cancer cell lines, assessed by density photometry. T means tumor, and N means adjacent normal tissues. * P<0.05 compared to MCF-7.
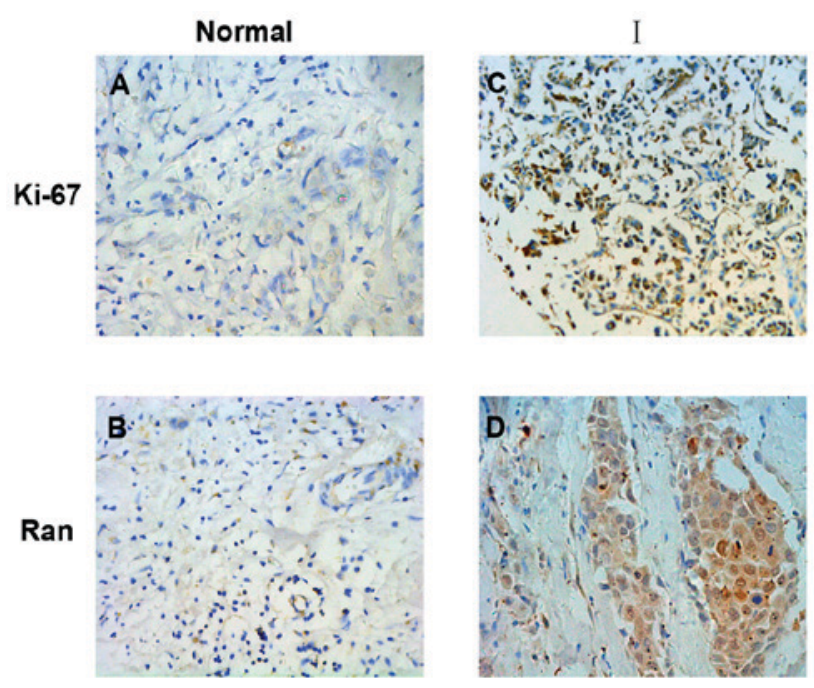
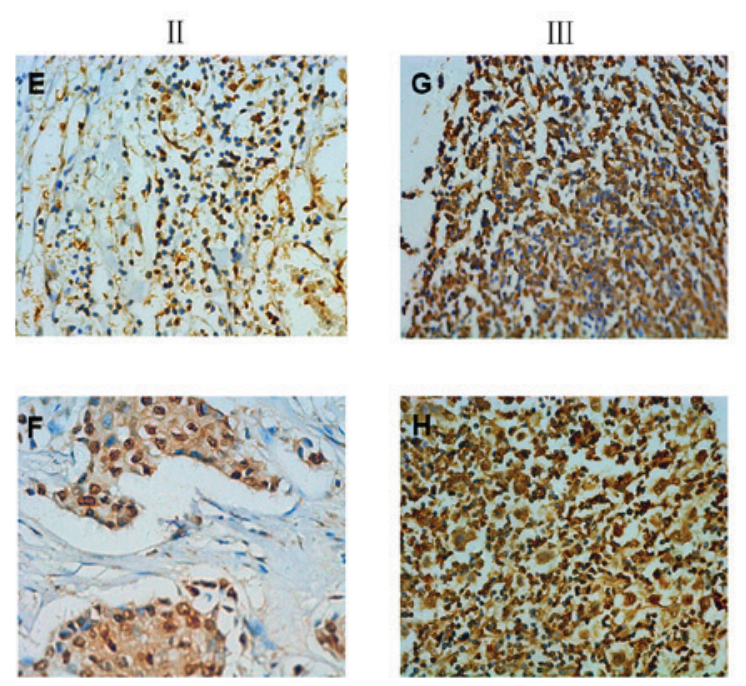

Figure 2. (A-H) Immunohistochemical staining of Ran and Ki-67 expression in paraffin-embedded breast cancer tissues. (A and B) Ran and Ki-67 staining in normal tissues. (C and D) Expression of Ran and Ki-67 was low in grade I (weakly positive) breast cancer tissues. (E and F) Expression of Ran and Ki-67 was moderate in grade II breast cancer tissues. (G and H) Expression of Ran and Ki-67 was high in grade III breast cancer tissues. The images in (A-H) were captured at x400 magnification.

three selected breast cancer cell lines that were analyzed by western blotting, the expression of Ran was found to be higher in the MDA-MB-231 cells compared to MCF-7 and SKBR-3 cells (Fig. 1C and D, $\mathrm{P}<0.05$ ). Therefore, in experiments that followed, we chose to use the MDA-MB-231 cell line.

To identify whether or not Ran expression was associated with breast cancer, immunohistochemistry was conducted to detect the expression and distribution of Ran and Ki- 67 proteins in paraffin-embedded mammary tissue sections obtained from 140 breast cancer patients including 109 patients with ductal breast cancer and 31 patients with others breast cancer types (e.g. lobular and papillary breast cancer). None of the patient included in this study were diagnosed with triple-negative breast cancer. The results showed that Ran was mainly located in the nuclear of cells and that high expression of Ran was significantly correlated with high expression of Ki-67 $(\mathrm{P}<0.05)$, which was also located in the cell nuclear (Fig. 2).

The relationships between Ran expression, clinicopathological characteristics and breast cancer patient survival. 
Table I. Ran, Ki-67 expression and clinicopathological parameters in 140 breast cancer specimens.

\begin{tabular}{|c|c|c|c|c|c|}
\hline & \multirow[b]{2}{*}{ Total } & \multicolumn{2}{|c|}{ Ran expression } & \multirow[b]{2}{*}{ P-value } & \multirow[b]{2}{*}{$\chi^{2}$} \\
\hline & & Low & High & & \\
\hline \multicolumn{6}{|c|}{ Age (years) } \\
\hline$\leq 50$ & 57 & 16 & 41 & 0.161 & 1.959 \\
\hline$>50$ & 83 & 15 & 68 & & \\
\hline \multicolumn{6}{|l|}{ Grade } \\
\hline I & 17 & 13 & 4 & $0.000^{\mathrm{b}}$ & 38.269 \\
\hline II & 60 & 14 & 46 & & \\
\hline III & 63 & 4 & 59 & & \\
\hline \multicolumn{6}{|l|}{ ER } \\
\hline Negative & 69 & 14 & 55 & 0.602 & 0.270 \\
\hline Positive & 71 & 17 & 54 & & \\
\hline \multicolumn{6}{|l|}{ PR } \\
\hline Negative & 70 & 14 & 56 & 0.541 & 0.372 \\
\hline Positive & 70 & 17 & 53 & & \\
\hline \multicolumn{6}{|l|}{ ErBb2 } \\
\hline Negative & 68 & 15 & 53 & 0.981 & 0.001 \\
\hline Positive & 72 & 16 & 56 & & \\
\hline \multicolumn{6}{|l|}{ Size } \\
\hline$\leq 2 \times 2 \times 2$ & 77 & 19 & 58 & 0.424 & 0.636 \\
\hline$>2 \times 2 \times 2$ & 63 & 12 & 51 & & \\
\hline \multicolumn{6}{|c|}{ Axillary lymph node status } \\
\hline No & 52 & 15 & 37 & 0.141 & 2.156 \\
\hline $\mathrm{Nx}$ & 88 & 16 & 72 & & \\
\hline \multicolumn{6}{|c|}{ Nerve invasion and metastasis } \\
\hline Negative & 85 & 25 & 60 & $0.010^{\mathrm{b}}$ & 6.631 \\
\hline Positive & 55 & 6 & 49 & & \\
\hline \multicolumn{6}{|c|}{ Vascular metastasis } \\
\hline Negative & 75 & 23 & 52 & $0.009^{\mathrm{b}}$ & 6.807 \\
\hline Positive & 65 & 8 & 57 & & \\
\hline \multicolumn{6}{|l|}{ Histology } \\
\hline Ductal & 109 & 26 & 83 & 0.360 & 0.835 \\
\hline Others & 31 & 5 & 26 & & \\
\hline \multicolumn{6}{|l|}{ Ki-67 } \\
\hline Low & 52 & 22 & 30 & $0.000^{\mathrm{b}}$ & 19.512 \\
\hline High & 88 & 9 & 79 & & \\
\hline
\end{tabular}

${ }^{a}$ Statistical analyses were performed by the Pearson $\chi^{2}$ test; ${ }^{b} \mathrm{P}<0.05$ was considered as statistically significant.

To further examine the pathophysiological significance of Ran, the relationships between its expression in breast cancer cells, the clinicopathological characteristics of the tumor, and patient survival, were investigated. The clinicopathological data are summarized in Table I. Expression of Ran in breast cancer cells was found to be markedly correlated with tumor grade $(\mathrm{P}=0.000)$, nerve invasion and metastasis $(\mathrm{P}=0.010)$, Vascular metastasis $(\mathrm{P}=0.009)$, and expression of Ki-67 ( $\mathrm{P}=0.000)$ (Table I). In contrast, there was no association between $\mathrm{Ran}$ and patient age $(\mathrm{P}=0.161)$, ER ( $\mathrm{P}=0.602), \mathrm{PR}(\mathrm{P}=0.541), \mathrm{ErBb} 2(\mathrm{P}=0.981)$, tumor size
$(\mathrm{P}=0.424)$, axillary lymph node status $(\mathrm{P}=0.141)$, or tumor histology $(\mathrm{P}=0.360)$. Kaplan-Meier survival curves showed that high expression of Ran was significantly associated with poor probability of overall survival (Fig. 3). Furthermore, univariate analysis showed that tumor grade $(\mathrm{P}=0.001)$, axillary lymph node status $(\mathrm{P}=0.000)$, nerve invasion and metastasis $(\mathrm{P}=0.003)$, vascular metastasis $(\mathrm{P}=0.000), \mathrm{Ki}-67$ expression $(\mathrm{P}=0.011)$ and Ran expression $(\mathrm{P}=0.002)$ were all significant prognostic factors of overall survival (Table II). Multivariate analysis using Cox's proportional hazards model indicated that Ran expression $(\mathrm{P}=0.023)$, tumor 
Table II. Survival status and clinicopathological parameters in 140 patients with breast carcinomas.

\begin{tabular}{|c|c|c|c|c|c|}
\hline & \multirow[b]{2}{*}{ Total } & \multicolumn{2}{|c|}{ Survival status } & \multirow[b]{2}{*}{ P-value ${ }^{a}$} & \multirow[b]{2}{*}{$\chi^{2}$} \\
\hline & & Alive & Dead & & \\
\hline \multicolumn{6}{|l|}{ Age (years) } \\
\hline$\leq 50$ & 57 & 21 & 36 & \multirow[t]{2}{*}{0.439} & \multirow[t]{2}{*}{0.597} \\
\hline$>50$ & 83 & 36 & 47 & & \\
\hline \multicolumn{6}{|l|}{ Grade } \\
\hline $\mathrm{I}$ & 17 & 13 & 4 & \multirow[t]{3}{*}{$0.001^{\mathrm{b}}$} & \multirow[t]{3}{*}{14.381} \\
\hline II & 60 & 27 & 33 & & \\
\hline III & 63 & 17 & 46 & & \\
\hline \multicolumn{6}{|l|}{ ER } \\
\hline Negative & 69 & 31 & 38 & \multirow[t]{2}{*}{0.317} & \multirow[t]{2}{*}{1.000} \\
\hline Positive & 71 & 26 & 45 & & \\
\hline \multicolumn{6}{|l|}{ PR } \\
\hline Negative & 70 & 26 & 44 & \multirow[t]{2}{*}{0.389} & \multirow[t]{2}{*}{0.73} \\
\hline Positive & 70 & 31 & 39 & & \\
\hline \multicolumn{6}{|l|}{ ErBb2 } \\
\hline Negative & 68 & 28 & 40 & \multirow[t]{2}{*}{0.913} & \multirow[t]{2}{*}{0.011} \\
\hline Positive & 72 & 29 & 43 & & \\
\hline \multicolumn{6}{|l|}{ Size } \\
\hline$\leq 2 \times 2 \times 2$ & 77 & 36 & 41 & \multirow[t]{2}{*}{0.107} & \multirow[t]{2}{*}{2.585} \\
\hline$>2 \times 2 \times 2$ & 63 & 21 & 42 & & \\
\hline \multicolumn{6}{|c|}{ Axillary lymph node status } \\
\hline N0 & 52 & 33 & 19 & \multirow[t]{2}{*}{$0.001^{\mathrm{b}}$} & \multirow[t]{2}{*}{17.734} \\
\hline $\mathrm{Nx}$ & 88 & 24 & 64 & & \\
\hline \multicolumn{6}{|c|}{ Nerve invasion and metastasis } \\
\hline Negative & 85 & 43 & 42 & \multirow[t]{2}{*}{$0.003^{\mathrm{b}}$} & 8.739 \\
\hline Positive & 55 & 14 & 41 & & \\
\hline Vascular m & & & & & \\
\hline Negative & 75 & 45 & 30 & $0.000^{\mathrm{b}}$ & 24.891 \\
\hline Positive & 65 & 12 & 53 & & \\
\hline Histology & & & & & \\
\hline Ductal & 109 & 46 & 63 & 0.501 & 0.451 \\
\hline Others & 31 & 11 & 20 & & \\
\hline Ki-67 & & & & & \\
\hline Low & 52 & 29 & 23 & $0.011^{\mathrm{a}}$ & 6.464 \\
\hline High & 88 & 27 & 61 & & \\
\hline Ran & & & & & \\
\hline Low & 31 & 20 & 11 & $0.002^{\mathrm{b}}$ & 9.345 \\
\hline High & 109 & 37 & 72 & & \\
\hline
\end{tabular}

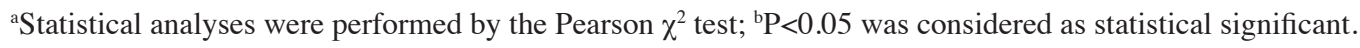

stage $(\mathrm{P}=0.002)$, axillary lymph node status $(\mathrm{P}=0.001)$, and vascular metastasis $(\mathrm{P}=0.032)$ were all independent prognostic indicators of overall survival (Table III). A positive correlation between Ran expression and $\mathrm{Ki}-67$ was found, with a correlation co-efficient of 0.834 ( $\mathrm{P}<0.01$, Fig. 3A). By using Kaplan-Meier analysis, patients with high expression level of Ran were significantly associated with short overall survival $(\mathrm{P}<0.01$, Fig. 3B).
Correlation of Ran with cell proliferation and its expression during the cell cycle. Since the expression of Ran had been found to be significantly correlated with the expression of $\mathrm{Ki}-67$, which is a known marker for cell proliferation in breast cancer specimens, this raised the hypothesis that Ran might play a role in cell cycle progression of breast cancer cells. We had already demonstrated that the expression of Ran was high in breast cells, especially in MDA-MB-231 
Table III. Contribution of various potential prognostic factors in 123 breast carcinomas specimens by Cox regression analysis.

\begin{tabular}{lccc}
\hline & Hazard radio & 95\% confidence interval & P-value \\
\hline Ran & 2.718 & $1.151-6.421$ & $0.023^{\mathrm{a}}$ \\
Ki-67 & 1.17 & $0.665-2.058$ & 0.586 \\
Age & 1.11 & $0.686-1.796$ & 0.67 \\
Histology & 0.848 & $0.491-1.467$ & 0.556 \\
Grade & 1.998 & $1.293-3.087$ & $0.002^{\mathrm{a}}$ \\
ER & 0.844 & $0.464-1.534$ & 0.578 \\
PR & 1.075 & $0.587-1.97$ & 0.814 \\
ErBb2 & 0.783 & $0.473-1.296$ & 0.341 \\
Size & 0.84 & $0.524-1.344$ & 0.466 \\
Axillary lymph node status & 3.697 & $2.088-6.544$ & $0.001^{\mathrm{a}}$ \\
Nerve invasion and metastasis & 1.186 & $0.723-1.946$ & 0.499 \\
Vascular metastasis & 1.756 & $1.049-2.939$ & $0.032^{\mathrm{a}}$ \\
\hline
\end{tabular}

Statistical analyses were performed by the log-rank test. ${ }^{\mathrm{a}} \mathrm{P}<0.05$ was considered as statistically significant.

A

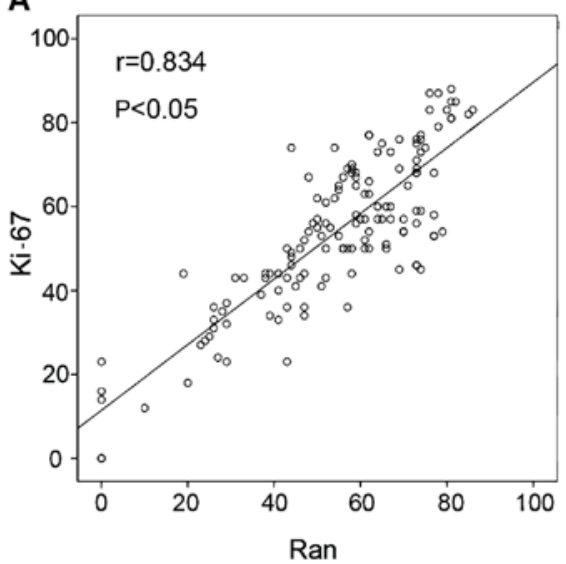

B

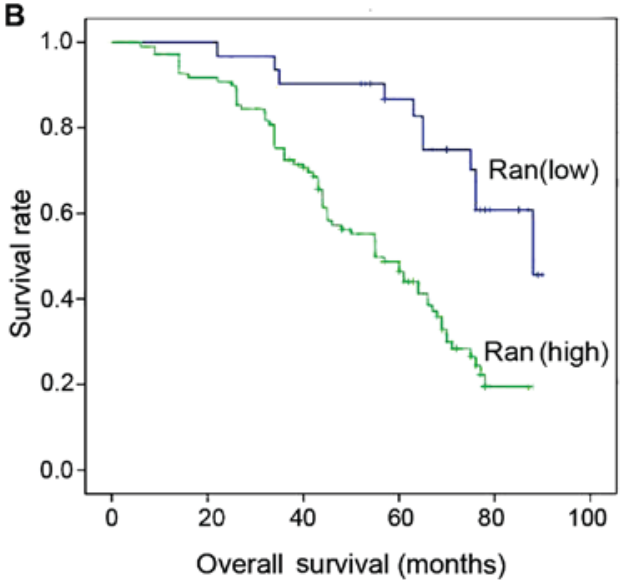

Figure 3. (A) Correlation between Ran GTPase (Ran) and Ki-67 expression in breast cancer. Scatter plot of Ran against Ki-67 with the regression line showing a correlation between them using Spearman's correlation co-efficient. The expression levels of these proteins from the immunostaining analysis, (B) Kaplan-Meier survival curves demonstrate the correlation between survival and the expression of Ran GTPase (Ran) in 140 patients with breast cancer. Patients with a lower Ran expression had a longer survival than those with a higher Ran expression.

cells (Fig. 1C). To confirm the involvement of Ran in the cell cycle, MDA-MB-231 cells were selected for a serum starvation and re-feeding experiment. Flow cytometry analysis revealed that after serum deprivation for $48 \mathrm{~h}, \mathrm{MDA}-\mathrm{MB}-231$ cells were arrested in the G1 phase as shown by an increase in the percentage of G1 phase cells to $76.39 \%$ of all cells. Upon re-feeding, cells were released from the G1 phase and the proportion of cells in the $\mathrm{S}$ phase gradually increased over time (Fig. 4A and B). To investigate whether the expression of Ran was cell cycle dependent, the proteins from the MDA-MB-231 cells were collected at different time-points within the cell cycle, for western blot analysis. Ran expression was found to be substantially increased in MDA-MB-231 cells as early as $4 \mathrm{~h}$ after serum re-feeding, and the expression of the cell proliferation marker PCNA showed a similar trend (Fig. 4C and D). Collectively, these results indicate that Ran may play an important role in cell proliferation of MDA-MB-231 cells.
Effect of Ran knock-down on cellular proliferation and the cell cycle. To further confirm the effect of Ran on the proliferation of breast cancer cells, chemically synthesized si-RNA was used to knock-down endogenous Ran in MDA-MB-231 before comparing cellular proliferation in these cells with that in cells transfected with negative control si-RNA. The results showed that Ran expression levels were decreased in MDA-MB-231 cells transfected with si-RNA of Ran $(\mathrm{P}<0.05)$, compared with cells transfected with negative control si-RNA (Fig. 5). Meanwhile, si-RNA\#2 achieved the most obvious knock-down efficiency (Fig. 5A and B). The levels of PCNA, cyclin A and cyclin E detected in Ran-si-RNA\#2 or control-si-RNA transfected MDA-MB-231 cells are shown in Fig. 5C. Then, methods combine EdU incorporation assay and CCK-8 assay were used to measure the proliferation of MDA-MB-231 cells after Ran shutdown. In the EdU incorporation assay, Ran shutdown leads to a remarkable reduce in the proportion of EdU-positive cells, indicating an restrained cell proliferation (Fig. 5D and E). 
A

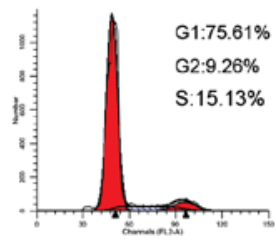

S48

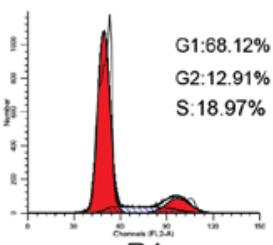

R4

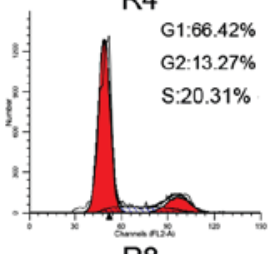

R8
C

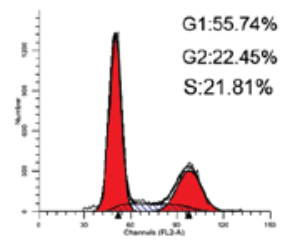

R12

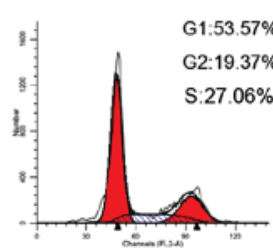

R24

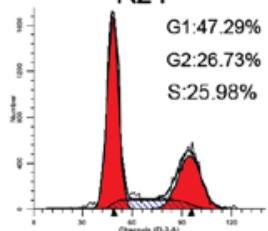

R48
B
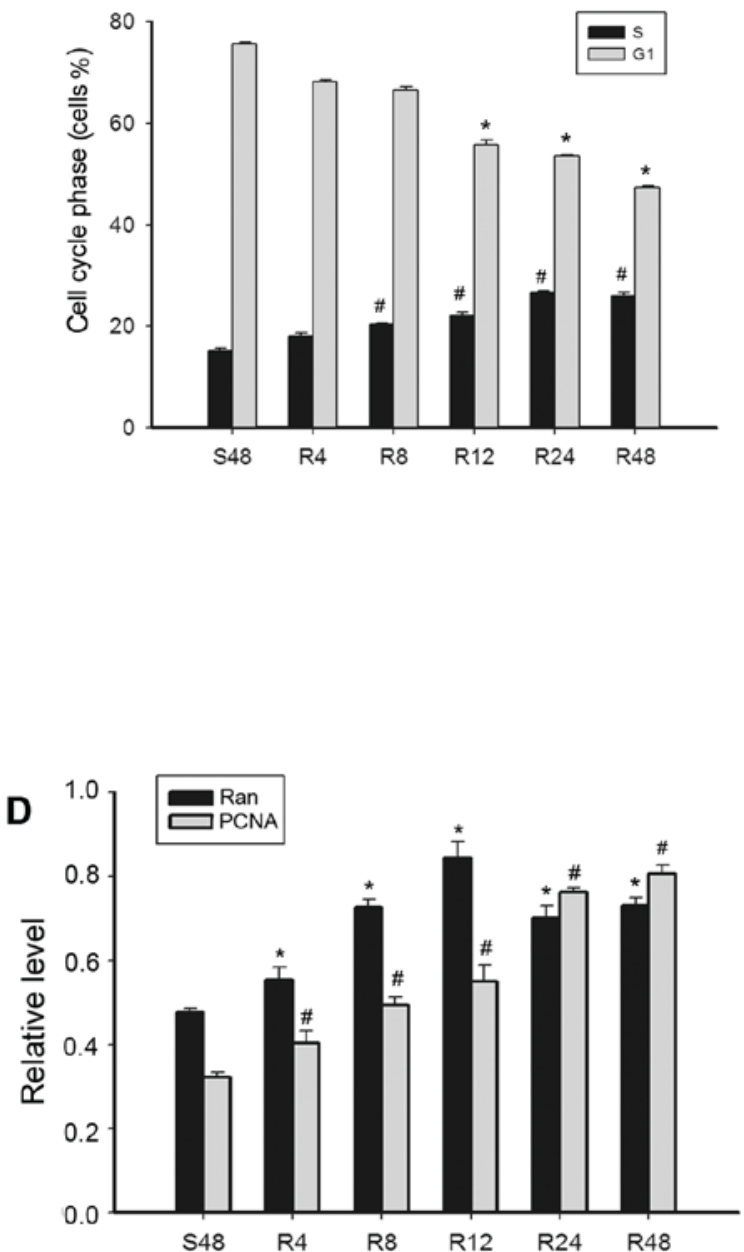

Figure 4. Expression of Ran and cell-cycle-related molecules detected in proliferating MDA-MB-231 cells by flow cytometry. (A and B) Cells synchronized at G1 progressed into the cell cycle when serum was added for S48, R4, R8, R12, R24, R48 h. (C and D) The S48 h MDA-MB-231 cells were released by re-feeding with serum, and cell lysates were prepared and analyzed by western blot analysis using antibodies against Ran, PCNA, and GAPDH (loading control). The bar chart plots the ratio of Ran, PCNA and GAPDH by densitometry. Data are means \pm SEM ${ }^{*, " P}<<0.05$ compared with control cells that were serum starved for $48 \mathrm{~h}$ (S48 h). SEM denotes standard error of the mean. S denotes serum starvation. R denotes serum release.

The CCK- 8 assay revealed that the rate of cell proliferation of MDA-MB-231 cells treated with si-RNA\#2 exhibited a significant decline compared with the cells treated with negative control si-RNA (Fig. 5F). Flow cytometry analyses of the cell cycle in MDA-MB-231 cells transfected with the active and control si-RNA confirmed these findings by showing a significant increase in the proportion of G1 phase cells and a concurrent decrease in the proportion of $\mathrm{S}$ phase cells, suggesting that down-regulated Ran had arrested the cell cycle of these cells (Fig. 5G). Taken collectively, these results indicate that the knock-down of Ran could result in tan inhibitory effect on cell proliferation associated with cell cycle arrest in MDA-MB-231 cells.

Association of Ran with breast cancer cell migration. The expression of Ran in breast cancer cells was found to be significantly correlated with nerve invasion and metastasis $(\mathrm{P}=0.010)$, and with vascular metastasis $(\mathrm{P}=0.009)$. On this basis it was hypothesized that Ran may play a role in the metastasis of breast cancer. Given that cell motility is indispensable for cancer metastasis, Transwell assays and wound healing assays were performed to determine the potential for Ran to induce cancer cell migration. Ran knock-down inhibited cell migration to the bottom chambers of the trans-wells compared to the control groups (Fig. 6A and B). The wound healing assays were conducted at different degrees of confluence for the control and si-RNA\#2 transfected cells. Representative photo-micrographs taken at 0,24 and $48 \mathrm{~h}$ after the cell surfaces were scratched in the wound healing assays are presented in Fig. 6C. These suggest that knock-down of of Ran could impede the wound healing process by resulting in a slower closure of the 'wound', scratched into a confluent epithelial mono layer (Fig. 6C).

To confirm this, a western blot analysis was performed to measure the levels of Ran protein in the control and active si-RNA transfected cells. It has been reported that E-cadherin and vimentin are markers of epithelial and mesenchymal cells, respectively. Therefore, we chose to analyze the levels of these proteins in the control-si-RNA and Ran-si-RNA\#2 infected cell groups. The expression of E-cadherin was found to be increased, while the expression of vimentin was decreased in the Ran-si-RNA\#2 infected cell groups (Fig. 6D).

Based on these results, it is hypothesized that knocking-down the expression of Ran could significantly inhibit breast cancer 
A

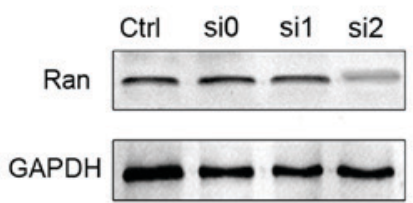

C

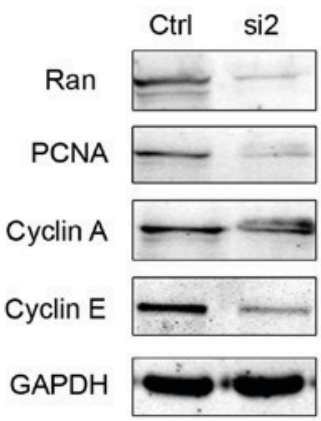

E

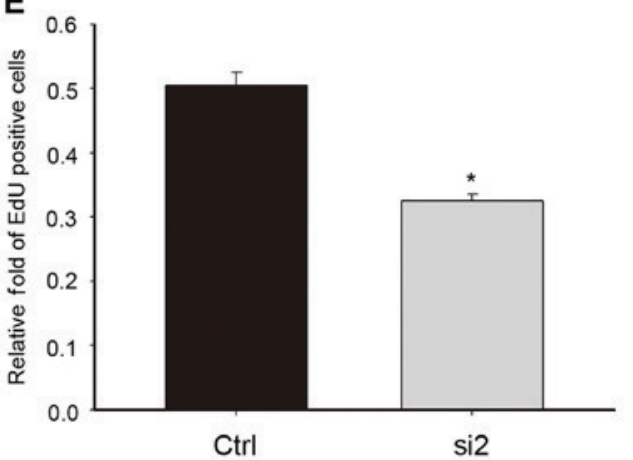

B

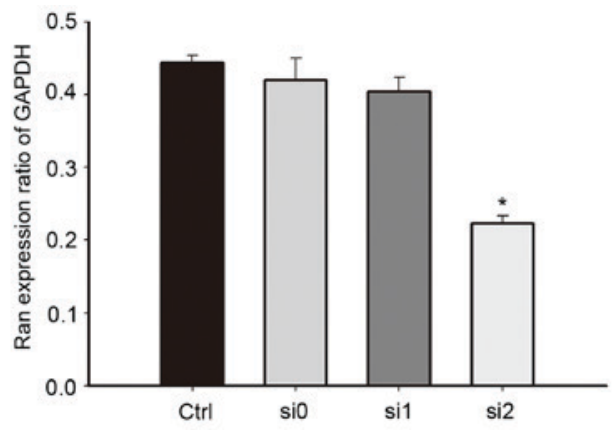

D

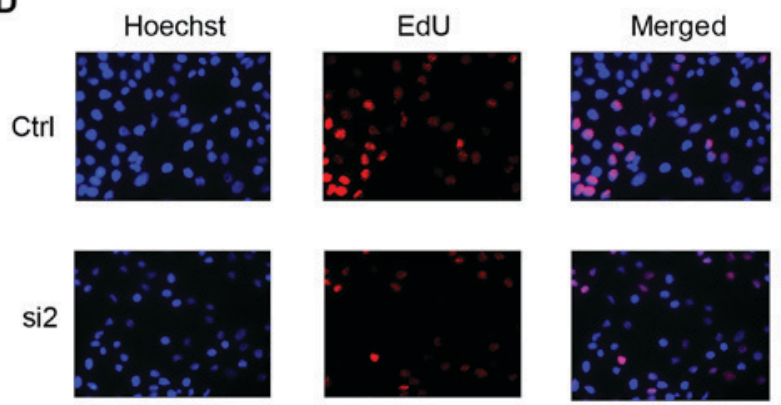

F

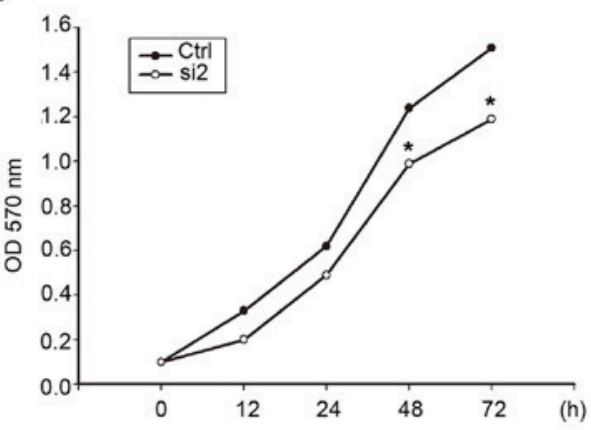

G
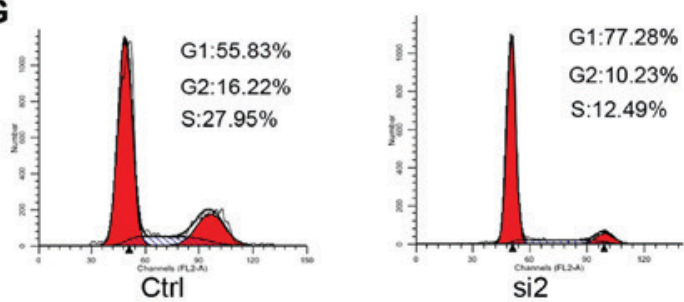

Figure 5. Ran knockdown inhibited MDA-MB-231 cell proliferation in vitro. (A) Expression of Ran protein in MDA-MB-231 cells as detected by western blotting $48 \mathrm{~h}$ after transfection. The transfected cells were subjected to a western blot analysis with antibodies against Ran and GAPDH (loading control). The data from each time-point were derived from three independent experiments. (B) The bar chart plots the ratio of Ran to GAPDH, as measured by densitometry. The data are presented as means $\pm \mathrm{SD},{ }^{*} \mathrm{P}<0.05$ compared to the control). (C) Western blot analysis of a series of cell cycle-related molecules, including PCNA, cyclin A and cyclin E, in MDA-MB-231 cells transfected with Ran-si-RNA\#2, and in control-Ran infected cells. GAPDH was used as a loading control. (D and E) EdU incorporation assay was analyzed by confocal microscopy in human MDA-MB-231 cells transfected with control siRNA and Ran siRNA\#2 (magnification, x20). (F) Cell proliferation as determined by the CCK-8 assay, which showed that Ran knock-down inhibited cell proliferation. The cell counting kit- 8 reagents were added to the medium and incubated for an additional $2 \mathrm{~h}$. The absorbance was measured at each of the indicated timepoints $(0,12,24,48$ and $72 \mathrm{~h})$. The data from each time-point were derived from three independent experiments. The data are presented as the means \pm SD, ${ }^{*} \mathrm{P}<0.05$ vs. control. (G) Ran expression was knocked down in the MDA-MB-231 cells transfected with Ran-si-RNA\#2. The adherent cells were collected and examined by flow cytometry, which showed a delay in the G1-S transition and significant arrest at G1 phase. The data are presented as means \pm SD. ${ }^{* *} \mathrm{P}<0.05$ compared to the control. The results are a combination of the data from three independent experiments.

cell migration by initiating the EMT process. Taken together, it is concluded that Ran is involved in breast cancer cell migration.

\section{Discussion}

With the prevalence of breast cancer having increased in recent decades, and it now being the second leading of cause of cancer-related deaths in women in developed countries (2), there is an urgent need for effective intervention therapies. Current treatments include surgery, chemotherapy, radiation therapy, hormone therapy and targeted therapy. Although there have been some improvements in diagnosis and the treatment breast cancer, in line with advances in medical technology, the prognosis of some breast cancers is still 
A

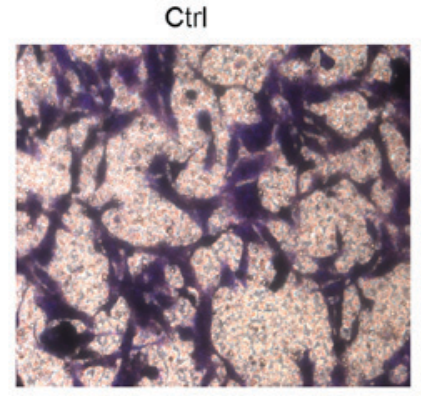

C

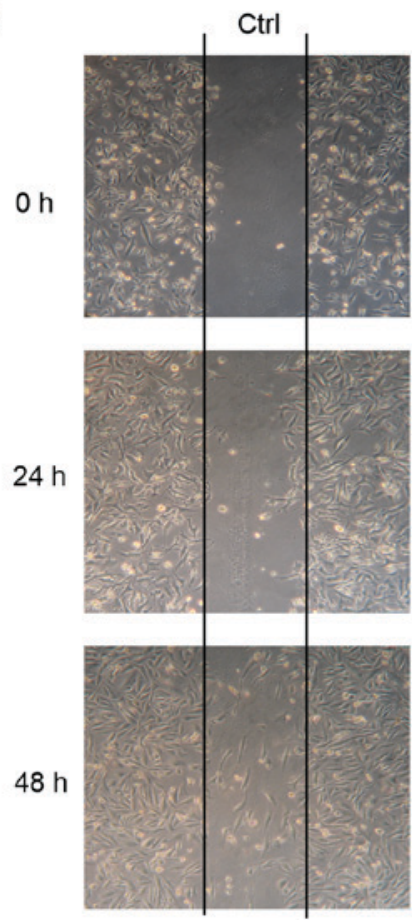

si2

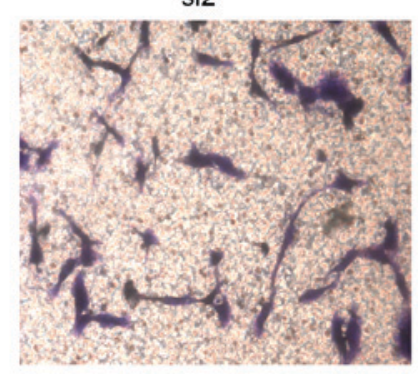

si2

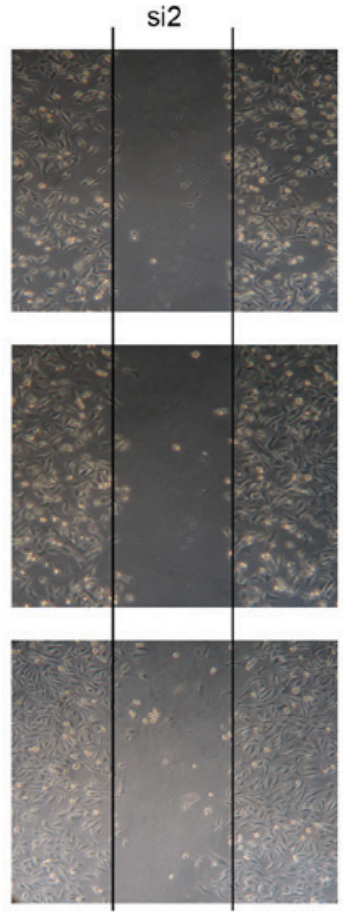

B

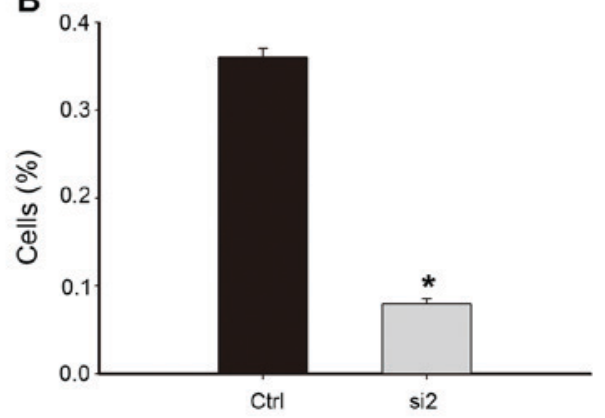

D

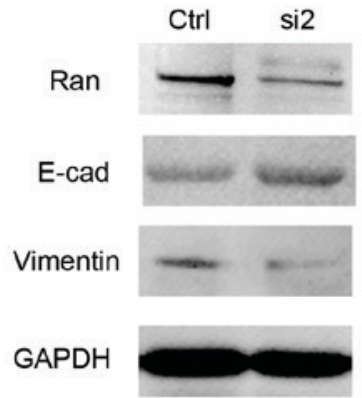

Figure 6. Effect of Ran knock-down on cell migration. (A) Ran knock-down inhibited cell migration in the transwell-assays. The control-si-RNA-transfected cells exhibited an increased ability to migrate through the membrane compared to the Ran-si-RNA\#2-transfected cells (magnification, $\mathrm{x} 40$ ). The data from each time-point were derived from three independent experiments. (B) The number of cells that migrated through the membrane was counted in 10 fields of view using a 40x objective lens. The data are presented as means $\pm \mathrm{SD},{ }^{*} \mathrm{P}<0.05$ compared to the control. (C) Wound healing assays with control-Ran and Ran-si-RNA\#2 transfected cell lines. The migration of the cells to the 'wound' was visualized at 0,24 and $48 \mathrm{~h}$ with an inverted Leica phase-contrast microscope (x200 magnification). (D) Western blot analysis of E-cadherin, vimentin and GAPDH (loading control) in the MDA-MB-231 cells transfected with control-Ran or Ran-si-RNA\#2. The levels of these tumor migration markers were significantly decreased in the cells in which Ran expression was knocked down. The data from each time-point were derived from three independent experiments. The results are presented as means \pm SD of three independent experiments.

poor (25), especially HER-2 positive or triple negative breast cancers (26). Therefore, a deeper understanding of the molecular events associated with breast cancer progression is urgently needed in order to inform the development of more effective treatments.

Genetic instability is a known hallmark of cancer. The Ran protein is a Ran small GTPase that has been implicated in the mitotic instability of cancer cells. Ran plays a number of physiological roles in the regulation of nuclear transport and has also been shown to play an active role in microtubule polymerization, mitotic spindle formation, and cytoskeleton organization during mitosis $(3,4,6)$. RanGTP-binding receptors, such as chromosome region maintenance 1 (CRM1) and importin- $\alpha / \beta$, control the transport of cargoes containing nuclear localization signals (NLS) or nuclear export signals (NES) (27).
Recent studies have demonstrated the over expression of Ran in multiple tumor types and that its expression is correlated with a poor patient prognosis, suggesting its importance in cell growth regulation. High levels of Ran in tumors have been associated with poor prognosis in renal cell carcinoma, breast cancer, and epithelial ovarian cancer $(15,17,28)$. Ran expression has been shown to be correlated with tumor grade, incidence of metastasis, and overall survival rates in renal cell cancer (15), and experimental silencing of Ran has been shown to decrease cell proliferation and cell adhesion in vitro (15). In human pancreatic cancer, down regulation of Ran expression in PANC-1 cells is associated with a reduction in Cyclin A, Cyclin D1, Cyclin E, CDK2, CDK4 and phospho-Rb protein levels compared with control and parental cells (29). In the present study, breast cancer tissue specimens were obtained from 140 cases and immunohistochemistry analyses were 
performed to determine the expression of Ran and Ki-67 in breast cancer tissue. The results demonstrated that high levels of Ran expression were related to the histological grade of the tumor, as well as to nerve invasion and metastasis, vascular metastasis and shorter survival probability.

In pancreatic cancer, expression of the androgen receptor (AR) and $G$ protein coupled receptor CXCR4 have been found to be significantly decreased following knock-down of Ran, indicating that Ran could regulate the invasion and metastasis of pancreatic cancer cells through effects on these receptors (30). In breast cancer, a few previous studies have similarly shown that Ran mediates the invasive functions of the prometastatic protein osteopontin (OPN) in breast cancer cells $(31,32)$. Over expression of Ran in benign mammary epithelium causes neoplastic transformation, dissemination and metastasis (15), and changes in Ran expression can lead to the abnormal transport of tumor oncogenes such as Akt or NF- $\mathrm{B}$ (19). Silencing Ran results in dysregulation of nucleocytoplasmic transport of transcription factors and down regulation of Mcl-1 expression, at the transcriptional level, which are reversed by inhibitors of the PI3K/Akt/mTORC1 and MEK/ERK pathways (33). These findings confirm that Ran is highly expressed in multiple tumor types, including breast cancer, and is associated with a more aggressive cell phenotype. In the present study, we performed si-RNA transfection into cancer cell lines obtained from cancerous breast tissue in order to knock-down the expression of Ran in vitro, and demonstrated that loss of Ran decreased the proliferation of MDA-MB-231 cells. Moreover, using trans-well and wound healing assays, we confirmed that Ran is also involved in breast cancer metastasis.

Current research suggests that Ran may not only be a useful prognostic biomarker, but may also be a potential anticancer therapeutic target. The association of Ran with metastasis increases its potential as a therapeutic target even further.

Both experimental and clinical evidence indicates that the aberrant activation of the epithelial-mesenchymal transition (EMT) of epithelial cells contributes to a range of pathological conditions, including cancer progression and metastasis (34). EMT promotes tumor progression by enhancing cancer cell migration and metastasis at distant sites, which is underlined by the loss of epithelial phenotype markers such as tight junctions, desmosomes, and cytoskeletal elements (35). In the present study, when Ran expression was knocked down, we observed that the expression of E-cadherin transmembrane proteins that are a marker of epithelial cells was increased, while the expression of vimentin, a marker of mesenchymal cells, was decreased. Thus, EMT was reduced in Ran knock-down cells. This indicates that EMT plays an important role in promoting the metastasis of breast cancer, mediated by Ran. However, whether Ran does indeed promote breast cancer metastasis through the EMT pathway, requires further validation. Since RanGTP levels can be regulated by serum and in particular by the growth factor HRG (17), it would of interest to determine whether changes in RanGTP levels in cancerous cells have an effect on the occurrence and development of breast cancer. The activation of Ran GTPase, and whether this promotes breast cancer metastasis, requires further study.

This study also has some disadvantages: Because of the large size of selected samples, and follow-up relevant error, the statistical result has certain bias. In addition, in the hunger to release experiment, the proliferation of tumor cell after release is not significant, this may be associated with cell activity, experimental conditions and so on. Finally, the $\mathrm{S}$ phase cells in the serum released $48 \mathrm{~h}$ have a slight decline than relative serum releases $24 \mathrm{~h}$, the corresponding Ran expression level also come down, this may be related to cell-contact-inhibition of cell proliferation to $48 \mathrm{~h}$, or cell survival space is limited and relative lack of nutrients and other factors.

Whilst the discovery of the involvement of the Ran protein in cancer progression is a significant finding, many aspects of its functional role remain to be elucidated, and it is not yet clear whether this knowledge will lead to an improvement in the diagnosis or treatment of cancer. Nevertheless, the rate of progress of research in this area has been rapid to date, and it is an exciting area of current interest for cancer biologists.

In conclusion, we have demonstrated that the Ran protein was highly expressed in breast cancer tissues and that knock-down of its expression inhibited the proliferation and migration of breast cancer cells in vitro. This may explain the mechanism of Ran over expression correlation with poor prognosis of breast cancer patients. Our findings also suggest that Ran may be a potentially effective target for future therapeutic interventions in the treatment of breast cancer.

\section{Acknowledgements}

This study was supported by the National Natural Science Foundation of China (no. 81672596), the Nan Tong Science and Technology Project (no. MS22015058) and the Nan Tong University Innovation Project (YKC15085).

\section{Competing interests}

The authors declare that they have no competing interests.

\section{References}

1. Parkin DM, Bray F, Ferlay J and Pisani P: Global cancer statistics, 2002. CA Cancer J Clin 55: 74-108, 2005.

2. Zhu L, Yang S, He S, Qiang F, Cai J, Liu R, Gu C, Guo Z, Wang C, Zhang W, et al: Downregulation of ubiquitin-specific protease 14 (USP14) inhibits breast cancer cell proliferation and metastasis, but promotes apoptosis. J Mol Histol 47: 69-80, 2016.

3. Dasso M: The Ran GTPase: Theme and variations. Curr Biol 12: R502-R508, 2002

4. Sazer S and Dasso M: The ran decathlon: Multiple roles of Ran. J Cell Sci 113: 1111-1118, 2000

5. Chafe SC, Pierce JB and Mangroo D: Nuclear-cytoplasmic trafficking of NTF2, the nuclear import receptor for the RanGTPase, is subjected to regulation. PLoS One 7: e42501, 2012.

6. Hetzer M, Bilbao-Cortés D, Walther TC, Gruss OJ and Mattaj IW: GTP hydrolysis by Ran is required for nuclear envelope assembly. Mol Cell 5: 1013-1024, 2000

7. Lonhienne TG, Forwood JK, Marfori M, Robin G, Kobe B and Carroll BJ: Importin-beta is a GDP-to-GTP exchange factor of Ran: Implications for the mechanism of nuclear import. J Biol Chem 284, 22549-22558, 2009.

8. Cimica V, Chen HC, Iyer JK and Reich NC: Dynamics of the STAT3 transcription factor: Nuclear import dependent on Ran and importin- $\beta 1$. PLoS One 6: e20188, 2011.

9. Doherty KJ, McKay C, Chan KK and El-Tanani MK: Ran GTPase as a target for cancer therapy: Ran binding proteins. Curr Mol Med 11: 686-695, 2011.

10. Segev N: Coordination of intracellular transport steps by GTPases. Semin Cell Dev Biol 22: 33-38, 2011.

11. Cook AG and Conti E: Nuclear export complexes in the frame. Curr Opin Struct Biol 20: 247-252, 2010. 
12. Saxena S, Gandhi A, Lim PW, Relles D, Sarosiek K, Kang C, Chipitsyna G, Sendecki J, Yeo CJ and Arafat HA: Ran GTPase and osteopontin in pancreatic cancer. Pancreat Disord Ther 3: $113,2013$.

13. Xia F, Lee CW and Altieri DC: Tumor cell dependence on Ran-GTP-directed mitosis. Cancer Res 68: 1826-1833, 2008.

14. Morgan-Lappe SE, Tucker LA, Huang X, Zhang Q, Sarthy AV, Zakula D, Vernetti L, Schurdak M, Wang J and Fesik SW: Identification of Ras-related nuclear protein, targeting protein for xenopus kinesin-like protein 2 , and stearoyl-CoA desaturase 1 as promising cancer targets from an RNAi-based screen. Cancer Res 67: 4390-4398, 2007.

15. Kurisetty VV, Johnston PG, Johnston N, Erwin P, Crowe P, Fernig DG, Campbell FC, Anderson IP, Rudland PS and El-Tanani MK: Ran GTPase is an effector of the invasive/metastatic phenotype induced by osteopontin. Oncogene 27: 7139-7149, 2008.

16. Muniyappa MK, Dowling P, Henry M, Meleady P, Doolan P, Gammell P, Clynes M and Barron N: MiRNA-29a regulates the expression of numerous proteins and reduces the invasiveness and proliferation of human carcinoma cell lines. Eur J Cancer 45 3104-3118, 2009.

17. Ly TK, Wang J, Pereira R, Rojas KS, Peng X, Feng Q, Cerione RA and Wilson KF: Activation of the Ran GTPase is subject to growth factor regulation and can give rise to cellular transformation. J Biol Chem 285: 5815-5826, 2010.

18. Ouellet V, Guyot MC, Le Page C, Filali-Mouhim A, Lussier C, Tonin PN, Provencher DM and Mes-Masson AM: Tissue array analysis of expression microarray candidates identifies markers associated with tumor grade and outcome in serous epithelial ovarian cancer. Int J Cancer 119: 599-607, 2006.

19. Abe H, Kamai T, Shirataki H, Oyama T, Arai K and Yoshida K: High expression of Ran GTPase is associated with local invasion and metastasis of human clear cell renal cell carcinoma. Int J Cancer 122: 2391-2397, 2008.

20. Tsai MY, Wiese C, Cao K, Martin O, Donovan P, Ruderman J, Prigent $\mathrm{C}$ and Zheng Y: A Ran signalling pathway mediated by the mitotic kinase Aurora A in spindle assembly. Nat Cell Biol 5 : 242-248, 2003.

21. Silljé HH, Nagel S, Körner R and Nigg EA: HURP is a Ran-importin beta-regulated protein that stabilizes kinetochore microtubules in the vicinity of chromosomes. Curr Biol 16: 731-742, 2006

22. Joukov V, Groen AC, Prokhorova T, Gerson R, White E, Rodriguez A, Walter JC and Livingston DM: The BRCA1/ BARD1 heterodimer modulates Ran-dependent mitotic spindle assembly. Cell 127: 539-552, 2006.

23. Yuen HF, Chan KK, Grills C, Murray JT, Platt-Higgins A, Eldin OS, O'Byrne K, Janne P, Fennell DA, Johnston PG, et al: Ran is a potential therapeutic target for cancer cells with molecular changes associated with activation of the PI3K/Akt/ mTORC1 and Ras/MEK/ERK pathways. Clin Cancer Res 18: 380-391, 2012.

24. Ning J, Liu W, Zhang J, Lang Y and Xu S: Ran GTPase induces EMT and enhances invasion in non-small cell lung cancer cells through activation of PI3K-AKT pathway. Oncol Res 21: 67-72, 2013.
25. Runowicz CD, Leach CR, Henry NL, Henry KS, Mackey HT, Cowens-Alvarado RL, Cannady RS, Pratt-Chapman ML, Edge SB, Jacobs LA, et al: American Cancer Society/American Society of clinical oncology breast cancer survivorship care guideline. CA Cancer J Clin 66: 43-73, 2016.

26. Li Y, Meeran SM,Patel SN, Chen H, Hardy TM and Tollefsbol TO: Epigenetic reactivation of estrogen receptor- $\alpha(\mathrm{ER} \alpha)$ by genistein enhances hormonal therapy sensitivity in ER $\alpha$-negative breast cancer. Mol Cancer 12: 9, 2013.

27. Görlich D, Kostka S, Kraft R, Dingwall C, Laskey RA, Hartmann E and Prehn S: Two different subunits of importin cooperate to recognize nuclear localization signals and bind them to the nuclear envelope. Curr Biol 5: 383-392, 1995.

28. Barrès V, Ouellet V, Lafontaine J, Tonin PN, Provencher DM and Mes-Masson AM: An essential role for Ran GTPase in epithelial ovarian cancer cell survival. Mol Cancer 9: 272, 2010.

29. Deng L, Lu Y, Zhao X, Sun Y, Shi Y, Fan H, Liu C, Zhou J, Nie Y, Wu K, et al: Ran GTPase protein promotes human pancreatic cancer proliferation by deregulating the expression of Survivin and cell cycle proteins. Biochem Biophys Res Commun 440: 322-329, 2013.

30. Deng L, Shang Y, Guo S, Liu C, Zhou L, Sun Y, Nie Y, Fan D, Lu Y and Guo X: Ran GTPase protein promotes metastasis and invasion in pancreatic cancer by deregulating the expression of AR and CXCR4. Cancer Biol Ther 15: 1087-1093, 2014

31. Dallol A, Hesson LB, Matallanas D, Cooper WN, O'Neill E, Maher ER, Kolch W and Latif F: RAN GTPase is a RASSF1A effector involved in controlling microtubule organization. Curr Biol 19: 1227-1232, 2009

32. Jeon H, Zheng LT, Lee S, Lee WH, Park N, Park JY, Heo WD, Lee MS and Suk K: Comparative analysis of the role of small $\mathrm{G}$ proteins in cell migration and cell death: Cytoprotective and promigratory effects of RalA. Exp Cell Res 317: 2007-2018, 2011.

33. Yuen HF, Chan KK, Grills C, Murray JT, Platt-Higgins A, Eldin OS, O'Byrne K, Janne P, Fennell DA, Johnston PG, et al: Ran is a potential therapeutic target for cancer cells with molecular changes associated with activation of the PI3K/Akt/ mTORC1 and Ras/MEK/ERK pathways. Clin Cancer Res 18: 380-391, 2012

34. Acloque H, Adams MS, Fishwick K, Bronner-Fraser $M$ and Nieto MA: Epithelial-mesenchymal transitions: The importance of changing cell state in development and disease. J Clin Invest 119: 1438-1449, 2009.

35. Foroni C, Broggini M, Generali D and Damia G: Epithelial mesenchymal transition and breast cancer: Role, molecular mechanisms and clinical impact. Cancer Treat Rev 38: 689-697, 2012. 